\title{
Left ventricular ejection fraction decrease after adenosine-induced stress as a predictor of ischemia
}

\author{
Gabriela Meléndez Ramírez, ${ }^{1}$ Edith Liliana Posada-Martínez, ${ }^{1}$ Aloha Meave-González, \\ ${ }^{1}$ Rafael Vera-Urquiza' and Juan Betuel Ivey-Miranda² \\ ${ }^{1}$ Secretaría de Salud, Instituto Nacional de Cardiología Ignacio Chávez, Department of Cardiovascular Magnetic Resonance; ${ }^{2}$ Instituto Mexicano
} del Seguro Social, Centro Médico Nacional Siglo XXI, Cardiology Hospital, Ciudad de México, Mexico

\begin{abstract}
Background: The decrease in left ventricular ejection fraction (LVEF) has been observed to be associated with three-vessel coronary disease in nuclear medicine studies; however, the role played by LVEF decrease has not been studied with cardiovascular magnetic resonance (CMR). Objective: To assess the association between LVEF decrease and cardiac ischemia in patients with CMR studies with adenosine. Method: Cross-sectional, comparative study. Inclusion criteria were: patients assessed with CMR with adenosine between January 2009 and June 2015. LVEF change was compared between patients testing positive for ischemia versus those who tested negative. Results: Fifty nine patients were included: 41 were males (70\%), mean age was $59.7 \pm 10.9$ years; $38 \%$ of the studies tested positive for ischemia. Delta LVEF (post-stress LVEF - resting LVEF) was $-0.16 \pm 5.9$ versus $5.3 \pm 4.7(p<0.001)$ in patients with and without ischemia, respectively. Conclusion: Patients who tested positive for ischemia had lower delta LVEF than those with negative studies for ischemia.
\end{abstract}

KEY WORDS: Cardiovascular magnetic resonance. Coronary artery disease. Left ventricular ejection fraction.

\section{Introduction}

Coronary artery disease (CAD) causes approximately $42 \%$ of all deaths related to cardiovascular conditions. ${ }^{1}$ In Mexico, heart disease is the leading cause of death. ${ }^{2}$ Invasive coronary angiography is the gold standard in coronary artery disease diagnosis, but it is not regarded as the initial study, and diagnosis can therefore be supported by functional tests (stress test or imaging stress test). ${ }^{3,4}$ Scintigraphy is a proven resource for myocardial effusion assessment; 4,5 it allows to simultaneously assess left ventricular perfusion, function and volumes. ${ }^{3}$ Post-stress and at rest perfusion images synchronized with electrocardiogram or gated-SPECT has enabled assessment of the post-ischemic stunning phenomenon; the magnitude of left ventricular ejection fraction (LVEF) post-stress depression with regard to rest is correlated with ischemia severity. Bestetti et al. ${ }^{4,5}$ reported that post-stress
LVEF significant reduction in gated-SPECT is due to end-systolic volume (ESV) increase caused by endocardial stunning. Currently, post-stress transient ischemic dilation and LVEF decrease are known to be important predictors of severe coronary artery disease in SPECT studies. ${ }^{6}$

Cardiac magnetic resonance (CMR) imaging is an increasingly used method owing to its high resolution, unlimited image planes and capability to provide different types of information in a single study; it is considered the gold standard for volumetric and functional quantification..$^{7-9}$ Perfusion quantification with CMR has shown good correlation with fractional flow reserve measures, the gold standard for the diagnosis of myocardial ischemia. ${ }^{8}$ As for ischemic heart disease, stress CMR can be used to detect ischemia-induced wall motion abnormalities; visual analysis enables observing areas of reduced perfusion, and with computational aid, identifying enhancement
Correspondence:

Edith Liliana Posada-Martínez

E-mail: liliposada1701@ gmail.com
Date of reception: 22-02-2017

Date of acceptance: 06-02-2018

DOI://dx.doi.org/10.24875/GMM.M18000147
Gac Med Mex. 2018;154:256-260

Contents available at PubMed www.gacetamedicademexico.com 
during the first-step phase, ${ }^{9,10}$ its diagnostic performance is comparable to that of other methods. ${ }^{11-13}$

In spite of the usefulness of adenosine CMR, there is no compelling information about the comparison of post-stress vs. rest functional parameters, as in other imaging techniques as, for example, lack of increase or decrease in post-stress LVEF (LVEF delta $\leq 0 \%$ ), which is associated with higher probability of severe CAD in SPECT.

\section{Method}

Comparative, cross-sectional study with non-random sampling of consecutive patients who underwent adenosine CMR for suspected or known CAD between January 2009 and June 2015. Demographic and clinical information was collected from patient charts. Patients were considered to have "known CAD" if they had a history of coronarography and angioplasty.

In this study, the reference standard for ischemic heart disease diagnosis was clear results consistent with myocardial ischemia on CMR.

CMR was performed with a 1.5-Tesla ${ }^{\circledR}$ equipment (Siemens Avanto, Erlangen, Germany) and a cardiac coil. Cine gradient-echo with cardiac synchronization was carried out during periods of inspiration apnea at rest. Adenosine was administered at $140 \mu \mathrm{g} / \mathrm{kg} / \mathrm{min}$ ute. At adenosine administration minute 3 , perfusion images were captured (T1-weighted first-step sequence), with gadolinium being administered at 0.1 $\mathrm{mmoL} / \mathrm{kg}$ body weight. Images were obtained on short axis at basal, mid and apical thirds. After the acquisition of images of perfusion under stress and with adenosine infusion, cine sequences under stress were acquired. Between 10 and 15 minutes later, images of perfusion at rest were obtained, with a second gadolinium dose being administered and, finally, late enhancement images were obtained 10 minutes later.

Left ventricular endo- and epicardial borders were delineated with the Argus program (semi-automatically and with manual correction when necessary) at end-diastole and end-systole, by means of which the ventricular function parameters were obtained both at rest and under stress.

Adenosine CMR-obtained volumetric variables were end-diastolic volume (EDV), ESV and LVEF at baseline and post-adenosine. LVEF delta was calculated with the following formula:

post-stress LVEF - rest LVEF
Table 1. Baseline characteristics of patients who underwent cardiac magnetic resonance imaging with adenosine for suspected or known CAD

\begin{tabular}{|c|c|c|c|c|c|c|}
\hline & \multicolumn{2}{|c|}{$\begin{array}{l}\text { Positive } \\
(n=22)\end{array}$} & \multicolumn{2}{|c|}{$\begin{array}{c}\text { Negative } \\
(\mathrm{n}=37)\end{array}$} & \multirow[t]{2}{*}{$\frac{\infty}{\delta}$} & \multirow[t]{2}{*}{$p$} \\
\hline & & SD & Me & SD & & \\
\hline \multirow[t]{2}{*}{ Age } & \multicolumn{2}{|c|}{$60 \pm 9.1$} & \multicolumn{2}{|c|}{$61 \pm 11.8$} & \multicolumn{2}{|c|}{0.740} \\
\hline & $n$ & $\%$ & $\mathrm{n}$ & $\%$ & (1) & \\
\hline Diabetes mellitus & 4 & 18.2 & 10 & 27 & & .440 \\
\hline Hypertension & 13 & 59 & 26 & 70.2 & & .380 \\
\hline Dyslipidemia & 4 & 18.2 & 10 & 27 & & .440 \\
\hline Smoking & 13 & 59 & 14 & 37.8 & 0 & .113 \\
\hline Known CAD & 14 & 64 & 6 & 16.2 & & 0.001 \\
\hline
\end{tabular}

$\mathrm{CAD}=$ coronary artery disease; patients were regarded as having CAD if they had known coronary artery disease or a history of coronarography or angioplasty.

Those responsible for LVEF delta calculation had no information on whether the patient had obtained a positive result for ischemia. In order to determine whether the study result was ischemia-positive or negative, images under stress were compared with those at rest. The presence of a defect under stress what wasn't found or that was smaller in size at rest was consistent with myocardial ischemia.

Descriptive analysis was carried out for quantitative variables with the mean and standard deviation or median and interquartile range, and with absolute and relative frequencies for qualitative variables. Qualitative variables comparative analysis was performed with Mann-Whitney's U-test or Student's t-test according to the distribution, and the comparison of percentages with the chi-square test or Fischer's exact test for frequencies expected of $<5$. Finally, a ROC-curve was plotted and specific points were calculated for coronary disease detection sensitivity and specificity. A p-value $<0.05$ was considered to be statistically significant. SPSS program, version 22 , was used.

\section{Results}

Fifty-nine patients were included, out of which 41 were males $(70 \%)$, and mean age was $59.7 \pm$ 10.9 years; $37 \%$ of studies were positive for ischemia. Baseline characteristics are shown in table 1. There was a higher percentage of patients with "known CAD" in the group of patients with positive studies in comparison with those who had negative studies $(p<0.001)$. 
Table 2. Comparison of ischemia results in patients with cardiovascular magnetic resonance imaging with adenosine

\begin{tabular}{|c|c|c|c|c|c|}
\hline & \multicolumn{2}{|c|}{ Positive $n=22$} & \multicolumn{2}{|c|}{ Negative $n=37$} & \multirow[t]{2}{*}{$p$} \\
\hline & Median & IQR & Median & IQR & \\
\hline Baseline LVEF & 58.3 & $48.2-65.7$ & 59 & $45.3-64$ & 0.940 \\
\hline Baseline ESV & 93 & 77.3-118.2 & 92.7 & 77.7-117.8 & 0.850 \\
\hline Baseline EDV & 37.6 & $22.1-59.8$ & 38 & $29-52.5$ & 0.860 \\
\hline Baseline SV & 50.3 & $39.6-59.5$ & 54.3 & $39.8-59.9$ & 0.620 \\
\hline \multirow[t]{2}{*}{ Mass } & 76.3 & $59-116.3$ & 88 & 56.3-112.7 & 0.730 \\
\hline & \multicolumn{2}{|c|}{ Mean \pm SD } & \multicolumn{2}{|c|}{ Mean \pm SD } & \\
\hline LVEF delta & \multicolumn{2}{|c|}{$-0.16 \pm 5.9$} & \multicolumn{2}{|c|}{$5.3 \pm 4.7$} & $<0.001$ \\
\hline EDV delta & \multicolumn{2}{|c|}{$15 \pm 14$} & \multicolumn{2}{|c|}{$7.1 \pm 10.4$} & 0.015 \\
\hline ESV delta & \multicolumn{2}{|c|}{$6.3 \pm 7.7$} & \multicolumn{2}{|c|}{$-1.5 \pm 7$} & $<0.001$ \\
\hline SV delta & \multicolumn{2}{|c|}{$8.6 \pm 10.4$} & \multicolumn{2}{|c|}{$9.3 \pm 7$} & 0.760 \\
\hline
\end{tabular}

$\mathrm{CMR}=$ cardiac magnetic resonance imaging, $\mathrm{EDV}=$ end-diastolic volume,

$\mathrm{ESV}=$ end-systolic volume, $\mathrm{SV}=$ stroke volume

The comparison of CMR results of patients with positive study versus ischemia-negative studies is shown in table 2. Baseline LVEF in positive-study patients was $58.3 \%(48.2-65.7)$ versus $59 \%(45.3-64)$ in patients with negative study $(p=0.94)$.

LVEF delta was significantly higher in patients with ischemia-positive studies in comparison to that in those with negative study $(-0.16 \pm 5.9$ versus $5.3 \pm$ 4.7 , respectively [ $<0.001])$. End-diastolic and end-systolic delta was significantly higher in those patients with ischemia-positive study.

Figure 1 depicts the comparison of LVEF delta between groups. No patient with ischemia-positive study had an LVEF delta $>7.6 \%$, while no patient with negative study had an LVEF delta lower than -2.4 $\%$. Overlapping of values was observed between positive and negative patients within the -2.4 and $7.6 \%$ range, where 24 of the 37 patients with ischemia-negative study and 15 of 22 with positive study were found.

Figure 2 depicts end-systolic volume comparison between groups. No patient with ischemia-negative study had an ESV delta $>15 \mathrm{~mL}$, whereas no patient with positive study had an ESV delta lower than $-5.7 \mathrm{~mL}$. Overlapping of values between positive and negative patients was observed within the 15 and $5.7 \mathrm{~mL}$ range, where 17 of the 22 patients with positive study were found. Figure 3 depicts the comparison of end-diastolic volume between groups. No patient with

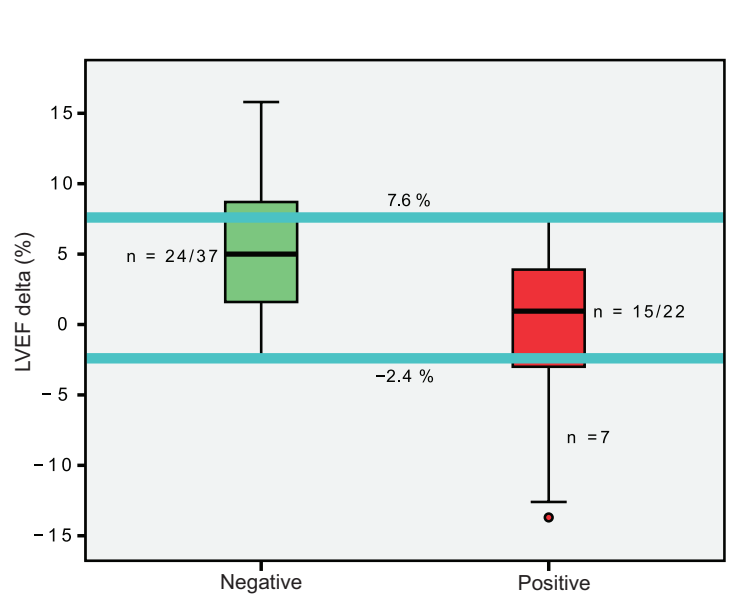

Figure 1. LVEF delta comparison in patients with ischemia-positive versus negative study. No patient with LVEF delta $>7.6 \%$ had myocardial ischemia-positive study and no patient with LVEF delta lower than $-2.4 \%$ had ischemia-negative study. LVEF = left ventricular ejection fraction.

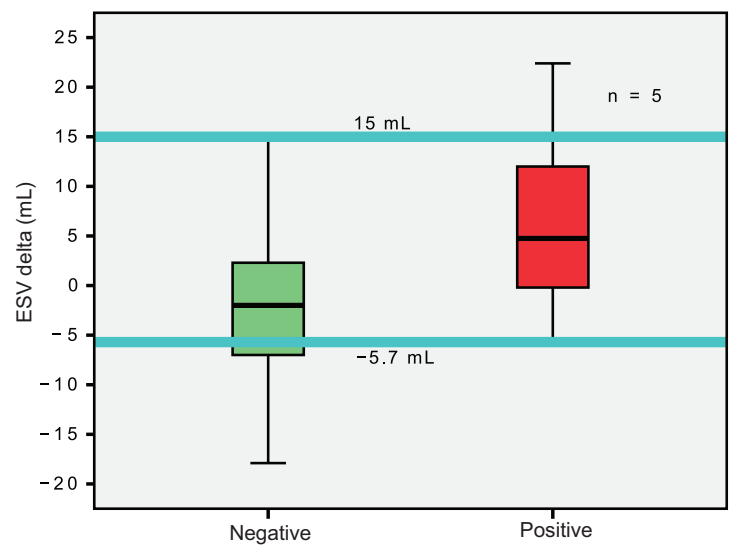

Figure 2. End-systolic volume comparison in patients with ischemia-positive versus negative study. No patient with ischemia-negative study had an ESV delta $>15 \mathrm{~mL}$. ESV = end-systolic volume.

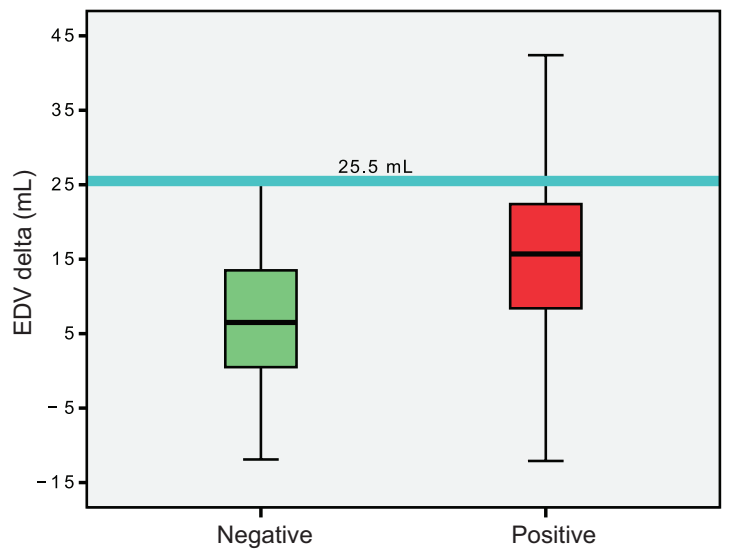

Figure 3. EDV delta comparison in patients with ischemia-positive versus negative studies. No patient with ischemia-negative study had an $E D V$ delta $>25.5 \mathrm{~mL}$. EDV = end-diastolic volume. 


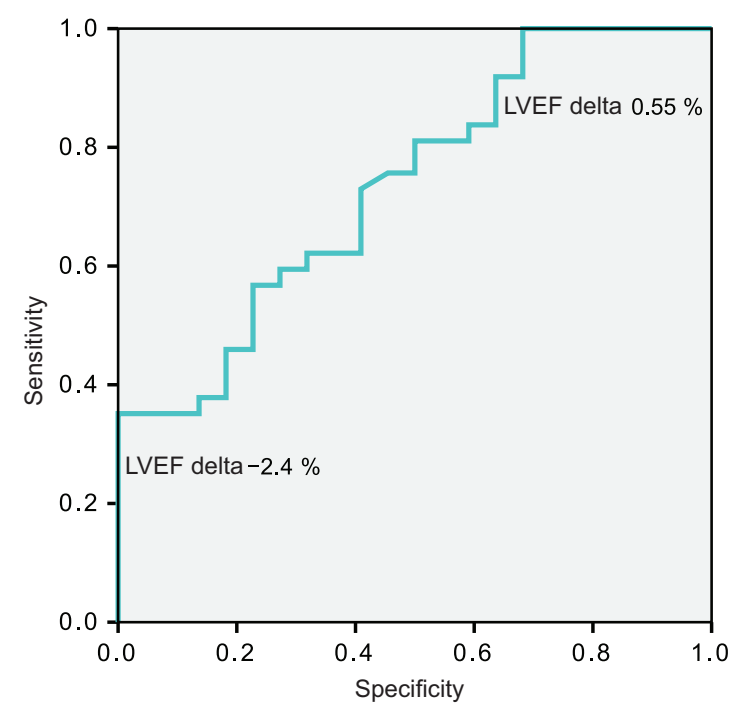

Figure 4. In the ROC curve, a LVEF delta cutoff value of $0.55 \%$ showed a sensitivity of $86 \%$ for the detection of ischemia-positive studies. The LVEF delta cutoff value of $-2.4 \%$ had a specificity of $100 \%$ for ischemia-positive studies. LVEF = left ventricular ejection fraction.

ischemia-negative study had an EDV delta $>25.5 \mathrm{~mL}$, while in the lower range they were very similar.

In the ROC curve (Fig. 4), sensitivity and specificity points of this test are established according to different cutoff values.

\section{Discussion}

There are several tests for the diagnosis of coronary artery disease, including nuclear medicine and magnetic resonance imaging. Perfusion defects assessed by these methods are useful for diagnosis and stratification of patients with ischemic heart disease. Since some time ago, stress-induced FEVI change on gated-SPECT ${ }^{6}$ has been considered a marker of more severe coronary artery disease, as reported by Bestetti et al. ${ }^{4,5}$, who also observed that this change is driven by left ventricle end-systolic volume increase.

Our main purpose was to know whether LVEF delta is different in patients with ischemia-positive versus negative studies assessed with adenosine CMR. The results were as expected, since statistically significant difference was found in LVEF delta by this method, in addition to confirming that there is also significant difference in ESV delta, as it has been described in specialized literature. ${ }^{7}$ Significant difference was also found in EDV. It is important mentioning that in our results there was LVEF delta overlapping, which ranged from 7.6 to $-2.4 \%$ in the analyses of patients with ischemia-negative versus positive studies, which in part might be explained by a LVEF decrease with the use of vasodilators in patients with ischemia-negative studies, as reported by Odagiri et al. ${ }^{14}$

In our opinion, this study is relevant, since a LVEF delta value lower than $-2.4 \%$ has very high specificity $(100 \%)$, which indicates that patients with a LVEF delta equal to or lower than this cutoff value have very high probabilities of having a positive study. On the other hand, a LVEF delta cutoff value $<0.55 \%$ has very high sensitivity $(86 \%)$ to detect positive studies, although low specificity. It is important pointing out that the main limitation of our study is that the results are not compared with the gold standard, coronary angiography.

If at first glance a study appears to be negative for ischemia, but a LVEF delta lower than $-2.4 \%$ is found, it must be cautiously analyzed, since it has very high probabilities of being positive; similarly, a study that initially appears to be positive, but has a LVEF delta $>0.55 \%$, it is highly probable for it to be negative for ischemia.

\section{Conclusions}

Patients with ischemia-positive studies have lower LVEF delta and higher ESV and EDV delta than those with negative studies. LVEF decrease after adenosine-induced stress on CMR can guide on the need for a diagnostic, and eventually therapeutic coronary angiography in patients with abnormal studies.

In our analysis we observed LVEF delta values overlapping in patients with ischemia-positive versus negative studies, which is why it is important for cutoff values to be used in order to increase sensitivity and specificity of the test.

\section{References}

1. Griffin BP, Kapadia SR, Rimmerman CM. The Cleveland Clinic Cardiology Board Review. USA: Lippincott Williams and Wilkins; 2013.

2. Instituto Nacional de Estadística, Geografía e Informática. Boletín de estadísticas vitales. México: Instituto Nacional de Estadística, Geografía e Informática; 2008.

3. Montalescot G, Sechtem U, Achenbach S, Andreotti F, Arden C, Budaj A, et al. 2013 ESC guidelines on the management of stable coronary artery disease: the Task Force on the management of stable coronary artery disease of the European Society of Cardiology. Eur Heart J. 2013;34:2949-3003.

4. Bestetti A, Triulzi A, Di-Leo C, Tagliabue L, Del-Sole A, Lomuscio A, et al. Myocardial scintigraphy by the gated SPECT method in coronary artery disease patients with post-ischemic stunning. G Ital Cardiol. 1999;29:143-148. 
5. Bestetti A, Di-Leo C, Alessi A, Triulzi A, Tagliabue L, Tarolo GL. Poststress end-systolic left ventricular dilation: a marker of endocardial post-ischemic stunning. Nucl Med Commun. 2001;22:685-693.

6. Gómez-Martínez MV, Ortega-Manrique A, Álvarez-Hernando J Theillac-Falcones B, De Jesús-Acosta M, Pereira-Del Moral R, et al. Disminución de la FEVI post-estrés en pacientes con cardiopatía isquémica y disfunción ventricular. Rev Esp Med Nucl Imagen Mol. 2014;33:160.

7. Lockie T, Ishida M, Perera D, Chiribiri A, De Silva K, Kozerke S, et al. High-resolution magnetic resonance myocardial perfusion imaging a 3.0-Tesla to detect hemodynamically significant coronary stenosis as determined by fractional flow reserve. J Am Coll Cardiol. 2011;57:70-75.

8. Pfeiffer MP, Biederman RW. Cardiac MRI: a general overview with emphasis on current use and indications. Med Clin North Am. 2015;99:849-861.

9. Selvanayagam JB, Kardos A, Francis JM, Wiesmann F, Petersen SE Taggart DP, et al. Value of delayed-enhancement cardiovascular magnetic resonance imaging in predicting myocardial viability after surgical revascularization. Circulation. 2004;110:1535-1541.

10. Jahnke C, Nagel E, Gebker R, Kokocinski T, Kelle S, Manka R, et al Prognostic value of cardiac magnetic resonance stress tests: adenosine stress perfusion and dobutamine stress wall motion imaging. Circulation. 2007;115:1769-1776.

11. Greenwood JP, Maredia N, Younger JF, Brown J, Nixon J, Everett C et al. Cardiovascular magnetic resonance and single-photon emission computed tomography for diagnosis of coronary heart disease (CEMARC): a prospective trial. Lancet. 2012;379:453-460.

12. Nandalur KR, Dwamena BA, Choudhri AF, et al. Diagnostic performance of stress cardiac magnetic resonance imaging in the detection of coronary artery disease: a meta-analysis. J Am Coll Cardiol. 2007;50(14):1343-1353.

13. Schwitter J, Wacker CM, Wilke N, Al-Saadi N, Sauer E, Huettle K, et al. MR-IMPACT II: Magnetic Resonance Imaging for Myocardial Perfusion Assessment in Coronary artery disease Trial: perfusion-cardiac magnetic resonance vs. single-photon emission computed tomography for the detection of coronary artery disease: a comparative multicenter, multivendor trial. Eur Heart J. 2013;34(10):775-781.

14. Odagiri K, Machii M, Tanaka T, Uehara A, Kurata C, Sakahara H, et al. Vasodilator stress impairs the left ventricular function obtained with gated single photon emission computed tomography in patients with known or suspected coronary artery disease. Circ J. 2010;74:2666-2673. 